\title{
Uber den Zusammenhang zwischen verschiedenen Messungen im Meer
}

\author{
Von Max Gillbricht \\ (Mit 1 Abbildung im Text)
}

Die klassische physikalische Meereskunde begnügte sich damit, das Seewasser durch Temperatur und Salzgehalt zu beschreiben und seine Bewegungsvorgänge aus diesen Grundgrößen zu berechnen oder mit Hilfe von Strömungsmessern direkt zur Anzeige zu bringen. Die hieraus abgeleiteten Vorstellungen über das Meer erwiesen sich jedoch im Laufe der Zeit als zu sehr vereinfacht, um so das gesamte Geschehen befriedigend erklären zu können. Verfeinerte Meßmethoden haben inzwischen gezeigt, wie viel heterogener Wassermassen sein können, als früher angenommen wurde. Jedoch verdanken wir diese Erkenntnisse nicht nur guten, z. T. über längere Zeiträume oder Strecken hinweg registrierenden Geräten zur Bestimmung von Temperatur, Salzgehalt und Strömung. Die ersten Hinweise in dieser Richtung stammen von ganz anderer Seite, denn viel länger bekannt ist die feine biologische Gliederung des Meeres, die vor allem für die praktische Fischerei von Bedeutung ist. So ist es naheliegend, zur Untersuchung der Feinstruktur auch biologische Indikatoren hinzuzuziehen. Allerdings lassen sich die Lebewesen selber nur mühsam und ungenau quantitativ erfassen, so daß indirekten Methoden gerne der Vorzug gegeben wird. Hierfür seien Chlorophyll, Trübung und Fluoreszenzstoffe nur als Beispiele genannt. Diese Aufzählung läßt sich noch erheblich erweitern, und es zeigt sich immer mehr, daß derartige Untersuchungen auch der physikalischen Meereskunde neue Erkenntnisse zu vermitteln vermögen (z. B. Joseph, 1959). Das älteste Verfahren dieser Art dürfte die Sauerstoffbestimmung sein. Sie läßt sich jedoch nur bedingt mit der für ozeanische Untersuchungen nötigen Genauigkeit durchführen. Vor allem bei Tiefenproben muß mit Zehrung innerhalb des Wasserschöpfers gerechnet werden (zur Hauptsache wohl durch Oxydation des Metalls bedingt), wenigstens werden die Schwankungen bei Parallelbestimmungen so ausgedeutet. Man muß aber daneben auch in Erwägung ziehen, daß der Sauerstoff vielleicht gar nicht so gleichmäßig verteilt ist. Diese Schwierigkeit besteht bei allen derartigen störanfälligen Messungen, daß sich die methodischen Streuungen nur schwer von den natürlichen trennen lassen. Dieser Nachteil beschränkt den Einsatz solcher Bestimmungsverfahren.

Nun ist aber auch die produktionsbiologische Forschung an derartigen Messungen interessiert und zum großen Teil für die Einführung dieser Methoden verantwortlich. Unter diesem Gesichtspunkt ist aber nicht nur die quantitative Verteilung von Wichtigkeit, jetzt treten vielmehr die biologische Bedeutung und der Zusammenhang der verschiedenen Messungen in den Vordergrund. Gerade der letzteren Frage dürfte eine größere Bedeutung zukommen. 
Die Zahl der untersuchten Komponenten und hierfür benutzten Methoden ist nicht mehr zu übersehen. Die Beobachtungen verschiedener Autoren lassen sich z. T. kaum noch vergleichen, und kein Bearbeiter ist mehr in der Lage, alle wünschenswerten Bestimmungen parallel durchzuführen.

Wie läßt sich nun über diese verwirrende Fülle eine Übersicht gewinnen? Es erscheint zunächst naheliegend, bei Versuchen in vitro jeweils nur einen Faktor zu variieren und seinen Einfluß auf die übrigen Komponenten zu bestimmen. Diese Methode ist für den Bereich des freien Wassers sicher noch nicht hinreichend angewandt worden, aber das hat seinen guten Grund, denn wir können diesen Lebensraum im Versuch künstlich nicht befriedigend nachahmen. Organismen und Partikel sinken zu Boden oder steigen auf, und die Spurenstoffe und die gelösten organischen Substanzen werden weitgehend an den Wandungen adsorbiert und bieten so den Bakterien hervorragende Entwicklungsmöglichkeiten. Die Umweltbedingungen sind folglich im Vergleich mit dem freien Wasser in mancher Hinsicht stark verändert, die biologischen Stoffumsetzungen dürften durchweg rascher und z. T. sicher auch anders verlaufen. Den unbeweglich am Boden liegenden Planktonorganismen sollte die ihnen im natürlichen Lebensraum gemäße ständige Wassererneuerung fehlen. Entsprechend unterscheiden sie sich dann auch bisweilen morphologisch oder physiologisch (z. B. Leuchtvermögen [Nordu, 1957]) von den natürlichen Populationen.

Derartige Überlegungen und Beobachtungen raten bei Experimenten zu besonderer Vorsicht. Es ist anzunehmen, daß sich zahlreiche Probleme im Glase kaum werden lösen lassen. Hierzu dürften z. B. organischer Detritus, gelöste organische Substanzen und deren natürlicher Abbau gehören. Bei Assimilation und Atmung der Planktonorganismen sollten bei hinreichender Vorsicht Versuche sehr wohl zu Erkenntnissen führen können, wie man sie in dieser Vollständigkeit im freien Wasser kaum wird erhalten können. Jedoch sollte es notwendig sein, diese Befunde in See nachzuprüfen.

Es erhebt sich mithin die Frage, in welchem Umfange lassen sich Aussagen über den Zusammenhang zwischen verschiedenartigen Messungen im Meere gewinnen? Welche Probleme lassen sich so direkt lösen, und bei welchen sind die Ergebnisse wenigstens hinreichend, die Realität experimenteller Befunde nachzuweisen? Das Problem liegt hierbei vor allem darin, daß sich eben alle Umweltfaktoren ständig ändern und entsprechend soweit möglich stets gemessen werden sollten. Es kommt also nicht so sehr auf lange Meßreihen einzelner Komponenten an als vielmehr darauf, mit recht vielen Beobachtern gleichzeitig möglichst viele Verfahren zur Anwendung zu bringen. Nur so dürften sich nach und nach die Beziehungen klären lassen.

Als Beispiel für diese Fragestellung seien hier einmal eine kurze Dauerstation mit dem Forschungskutter "Uthörn“ am 5. August 1959 unmittelbar unter der Helgoländer Düne und eine Station in der Tiefen Rinne behandelt.

Es wurden auf dieser Fahrt folgende Komponenten bestimmt:

1) Tiefe der Probennahme $[\mathrm{m}]$

2) Wassertemperatur $\left[{ }^{0} \mathrm{C}\right]$

3) Salzgehalt [\%o] durch Cl-Titration (ausgeführt vom Deutschen Hydrographischen Institut, Hamburg)

4) Kalziumgehalt $[\mathrm{mg} / 1]$ durch Titration mit ADTE mit Murexid als Indikator

5) $\triangle \mathrm{EK}\left[\lg \frac{\mathrm{I}_{0}}{\mathrm{I}} / \mathrm{m}\right]$ bezogen auf den Luftwert mit einem registrierenden Trübungsmesser mit einer Meßstrecke von $1 \mathrm{~m}$ 
6) Gehalt an (zumeist gelöster) organischer Substanz durch Oxydation mit alkalischer Permanganatlösung und Angabe als C-Aquivalent [mg C/1] (GrLbrichr, 1957)

7) Phytoplankton [mg C/m $/ \mathrm{m}^{3}$ ] durch Auszählung kleiner Wasserproben (Grllbricht, 1959) und Umrechnung auf $\mathrm{C}$-Gehalt

8) Zooplankton [ $\mathrm{mg} \mathrm{C} / \mathrm{m}^{3}$ ] Bestimmung wie Phytoplankton

9) "Rest" $\left[\mathrm{mg} \mathrm{C} / \mathrm{m}^{3}\right]$ offensichtliche Zersetzungsprodukte des Planktons; Bestimmung wie Phytoplankton

Durch Herrn Prof. Kalle (Deutsches Hydrographisches Institut)1) wurden gemessen:

10) Feintrübung [EK $10^{-3} / 5 \mathrm{~cm}$ ] mit dem Elko II und Filter $\mathrm{S} 75 \mathrm{E}$

11) Gelbstoff [EK $10^{-3} / 5 \mathrm{~cm}$ ] mit dem Elko II (Extinktion Filter S $42 \mathrm{E}$ - Extinktion Filter S $75 \mathrm{E}$ )

12) Fluoreszenzstoff [Einheiten] gemäß Kalde (1951) unter Benutzung eines Schott-Filters GG 18 als Zwischenstandard

13) Organismische Fluoreszenz [Einheiten] gemäß KALLE (1951) unter Zwischenschaltung eines Schott-Filters L 3

14) Chlorophyll $\left[\mathrm{mg} / \mathrm{m}^{3}\right]$ nach KaLlE (1951)

15) Phosphat [mg Phosphat-P/m $\left./ \mathrm{m}^{3}\right]$ nach Kalle (1934)

Durch Herrn Dr. Gunkel (Biologische Anstalt Helgoland) ${ }^{1}$ ) wurde bestimmt:

16) Bakterienzahl [Anzahl $/ \mathrm{ml}]$

Die Proben wurden unmittelbar vor denjenigen für die hydrographischen und Planktonbestimmungen mit einem modifizierten JZ-Schöpfer (ZoBELL, 1941) entnommen. Dieses Gerät gestattet die Gewinnung von Wasserproben unter sterilen Bedingungen. Die Anlage der Kulturen wurde nach der Gußplattenmethode sofort an Bord durchgeführt, und zwar unter Verwendung des Seewasser-Nährbodens 2216 (ZoBELL, 1946). Die beimpften Petrischalen wurden bei $18^{\circ} \mathrm{C}$ bebrütet und nach zwölf Tagen mit Hilfe eines Zählpultes mit Ringleuchte und Vergrößerungsglas ausgezählt. Die Methode erfaßt heteratroph-aerobe Bakterien, die in der Lage sind, auf dem komplexen Pepton-Nährboden zu wachsen. Es wurden jeweils mehrere Parallelplatten angelegt.

Insgesamt stehen 29 entsprechende Parallelmessungen zur Verfügung. Drei Trübungs- und zwei Phosphatwerte mußten durch Interpolation ergänzt werden. Bei den weiteren Rechnungen werden die oben gebrachten Dimensionen benutzt, auf deren Angabe jedoch verzichtet wird, um so die Gleichungen besser lesbar zu machen.

In den Gleichungen werden folgende Abkürzungen verwandt:

$\begin{array}{llll}\mathrm{m} & \text { für Tiefe der Probennahme } & \text { Rest } & \text { für Rest } \\ \mathrm{t} & \text { für Wassertemperatur } & \text { Feintr } & \text { für Feintrübung } \\ \mathrm{S} & \text { für Salzgehalt } & \text { Gelbst } & \text { für Gelbstoff } \\ \mathrm{Ca} & \text { für Kalziumgehalt } & \text { Fl } & \text { für Fluoreszenzstoff } \\ \triangle \mathrm{EK} & \text { für } \triangle \mathrm{EK} & \text { org Fl } & \text { für organismische Fluoreszenz } \\ \mathrm{C} & \text { für organische Substanz } & \text { Chl } & \text { für Chlorophyll } \\ \text { Phytopl für Phytoplankton } & \mathrm{P} & \text { für Phosphat } \\ \text { Zoopl } & \text { für Zooplankton } & \text { Bakt } & \text { für Bakterienzahl }\end{array}$

Zunächst werden die Korrelationskoeffizienten zwischen sämtlichen Verfahren bestimmt ${ }^{2}$ ). Diese Werte sagen aus, wie eng die Schwankungen zweier Meßgrößen miteinander verbunden sind, wobei sich diese gleichsinnig (positive Korrelation) oder gegensinnig (negative Korrelation) ändern können. Der Wert liegt zwischen + oder -1 (vollständige Korrelation) und 0 (keine Korrelation). Aus dem Korrelationskoeffizienten und der Anzahl der Messungen läßt sich

1) Herrn Prof. Kalle und Herrn Dr. Gunkel danke ich für die Überlassung der Meßswerte, der Deutschen Forschungsgemeinschaft für das Elko II (Zeiss),

2) Hinsichtlich der praktischen Durchführung der hier erwähnten Berechnungen sei auf die einschlägige statistische Literatur verwiesen.

Für die vorliegende Untersuchung wurden folgende Werke benutzt:

Goulden, C., 1952: Methods of Statistical Analysis. New York.

Grbelein, H., und Herte, H., 1951: Statistische Urteilsbildung. Springer-Verlag, Berlin.

RaUen, H., 1956: Biochemisches Taschenbuch. Springer-Verlag, Berlin. 
abschätzen, mit welcher Wahrscheinlichkeit eine Korrelation im angegebenen Sinne besteht. Die bekannte $\mathbf{t} / \mathrm{S}$-Beziehung sieht in unserm Falle und als Korrelationskoeffizient geschrieben so aus:

$$
\mathrm{r}_{\mathrm{t.S}}=-0,988 \text { Wahrscheinlichkeit } \gg 99,9 \%
$$

hieraus folgt: $t=98,32-2,540 \mathrm{~S}$

Der mittlere Fehler zwischen der gemessenen und der so berechneten Temperatur beträgt $\pm 0,11^{0}$, während die Streuung lediglich auf Grund der mittleren Fehler von t- und S-Messungen nur etwa halb so groß sein dürfte.

Es wäre nun denkbar, daß die Temperatur nicht nur vom Salzgehalt, sondern auch von der Tiefe der Probenentnahme abhängt. Nehme ich eine Mehrfachkorrelation dieser Art an, so bekomme ich hierfür einen gemeinsamen Korrelationskoeffizienten von (ohne Vorzeichen):

Die Gleichung lautet dann:

$$
\mathrm{R}_{\mathrm{t.S}, \mathrm{m}}=0,989 \text { Wahrscheinlichkeit } \gg 99,9 \%
$$

$$
t=93,38-2,383 \mathrm{~S}-0,0008 \mathrm{~m}
$$

Die Temperaturabnahme mit der Tiefe ist nur gering, und der Korrelationskoeffizient wird daher auch nur unwesentlich verbessert. Viel interessanter ist in diesem Zusammenhang die Frage, wie groß sind die "partiellen" Korrelationskoeffizienten? Darunter versteht man die Beziehungen zwischen Temperatur und Salzgehalt bzw. Tiefe unter der Voraussetzung, daß jeweils die andere Variable konstant gehalten wird, d. h. also, wie würde die Temperatur mit dem Salzgehalt bzw. der Tiefe korreliert sein, wenn die Tiefe bzw. der Salzgehalt konstant blieben? Die betreffenden Werte sind:

$$
\begin{aligned}
& \mathrm{r}_{\mathrm{t}, \mathrm{S} \cdot \mathrm{m}}=-0,974 \\
& \mathrm{r}, \mathrm{m} \cdot \mathrm{s}=-0,367
\end{aligned}
$$

$$
\begin{gathered}
\text { Wahrscheinlichkeit } \\
\geqslant 99,9 \% \\
\angle 95 \%
\end{gathered}
$$

Die Temperaturabnahme mit der Tiefe ist also nicht hinreichend gesichert, wenn wir sie unabhängig vom Salzgehalt betrachten.

Entsprechende Überlegungen lassen sich auch hinsichtlich des Salzgehalts aufstellen. Hier ergibt sich nun nach Eliminierung von t:

$$
\mathrm{rs}_{\mathrm{s}, \mathrm{m} . \mathrm{t}}=-0,021
$$

Diese partielle Korrelation ist also praktisch bedeutungslos, so daß man zweckmäßig aus $r_{\text {t.s }}$ bestimmt:

$$
\mathrm{S}=38,54-0,384 \mathrm{t}
$$

Diese ganzen Überlegungen besagen im Grunde genommen nur, daß die $\mathrm{t} / \mathrm{S}$ Relation streng gültig ist. Wir haben es also mit einem Gebiet zu tun, in dem sich zwei Wassermassen mit definierter Temperatur und definiertem Salzgehalt mischen. Eine Schichtung ist praktisch nicht vorhanden. Dies läßt sich auch dadurch zeigen, daß keine merkliche Verbesserung eintritt, wenn bei der Tiefenverteilung mit Gleichungen höheren Grades gearbeitet wird.

Beim vorhergehenden Problem war der Weg vielleicht etwas umständlich, aber es sollte an diesem einfachen Beispiel nur gezeigt werden, in welcher Weise die schwierigen Fragen angegriffen werden sollen. Bei den übrigen Komponenten liegen die Dinge ungünstiger. Zunächst sind die Messungen relativ unsicher, so daß mit sonderlich guten Korrelationskoeffizienten schon aus diesem Grunde nicht zu rechnen ist. Als Maß hierfür ist in der folgenden Tabelle angegeben, mit wieviel Prozent die ( $z$. T. geschätzte und daher recht unsichere) mittlere Streuung der Einzelmessungen an der beobachteten mittleren Streuung aller Meßwerte beteiligt ist. Ferner werden der Meßfehler und die bei den Mehrfachkorrelationen verbleibende Streuung der Meßwerte gebracht. 


\section{Tabelle 1}

\begin{tabular}{|c|c|c|c|}
\hline Art der Messung & $\begin{array}{l}\text { bekannter od. } \\
\text { geschätzter } \\
\text { Meßfehler in } \\
\text { Einheiten }\end{array}$ & $\begin{array}{l}\text { Anteil der } \\
\text { methodischen } \\
\text { Streuung an } \\
\text { der Gesamt- } \\
\text { streuung in } \%\end{array}$ & $\begin{array}{l}\text { Reststreuung } \\
\text { bei Mehrfach- } \\
\text { korrelation } \\
\text { in } \% \text { der Ge- } \\
\text { samtstreuung }\end{array}$ \\
\hline Tiefe & 0,2 & 2 & - \\
\hline Temperatur & 0,02 & 3 & 15 \\
\hline organismische Fluoreszenz & 0,3 & 3 & 79 \\
\hline Salzgehalt & 0,02 & 7 & 15 \\
\hline Chlorophyll & 0,2 & 9 & 67 \\
\hline$\triangle \mathrm{EK}$ & 0,01 & 12 & 35 \\
\hline Feintrübung & 0,5 & 19 & 70 \\
\hline Fluoreszenz & 0.3 & 25 & 57 \\
\hline org. Subst. & 0,1 & 26 & 75 \\
\hline Zooplankton & 10 & 30 & 78 \\
\hline Gelbstoff & 0,5 & 35 & 71 \\
\hline Phosphat & 0,3 & 43 & 82 \\
\hline Bakterien & 30 & 43 & 73 \\
\hline Kalzium & 4 & 57 & 78 \\
\hline Phytoplankton & 10 & 84 & 75 \\
\hline Rest & 10 & 91 & 78 \\
\hline
\end{tabular}

Es läßt sich nur schwer sagen, womit sich die Komponenten gut werden korrelieren lassen. In der Praxis wurde daher so vorgegangen, daß zunächst direkt die höheren Korrelationskoeffizienten herausgesucht wurden und dann diejenigen, die erst nach Eliminierung der hydrographischen Daten $(t, S, m)$ zum Vorschein kamen. Werden nun mit Hilfe dieser Komponenten Mehrfachkorrelationen ermittelt, so läßt sich bestimmen, welche Messungen für die Beschreibung der Verteilung der gerade untersuchten Substanz von Bedeutung sind. Die betreffenden Ergebnisse werden dann hier gebracht.

Beginnen wir mit dem Chlorophyll. Es wird gerne als Maß für das Phytoplankton benutzt. Prüfen wir diese Beziehung nach, so ergibt sich:

$$
\mathrm{Chl}=3,74+0,0711 \text { Phytopl }
$$

Hieraus errechnet sich ein Chlorophyllgehalt des Phytoplanktons (bezogen auf dessen C-Gehalt) von 7,11\%. Dieser Wert liegt innerhalb der aus der Literatur bekannten Daten (Krex, 1958). Im Mittel sind nur etwa 35\% des Chlorophylls an das lebende Plankton gebunden. Diese Tatsache ist schon bekannt (GILlbRicht, 1952). Sie besagt übrigens keinesfalls, daß Chlorophyllmessungen nicht den ihnen zugeschriebenen Wert besitzen. Bei regionalen Betrachtungen wird es vielmehr stets so sein, daß in planktonreichen Gegenden auch viel Chlorophyll an Detritus gebunden ist, und erst bei näherer Betrachtung treten die soeben geschilderten Verhältnisse störend in Erscheinung. Aber es erhebt sich jetzt natürlich die Frage, ob sich durch Hinzuziehung weiterer Komponenten die Verteilung des Chlorophylls besser beschreiben läßt. Als zweckmäßig erwies sich eine Mehrfachkorrelation mit Salzgehalt, Phosphatgehalt und organismischer Fluoreszenz:

Die Gleichung lautet dann:

$$
\mathrm{R}_{\text {Chl.S, P, org } \mathrm{FI}}=0,7438 \quad \text { Wahrscheinlichkeit } \gg 99,9 \%
$$

$$
\mathrm{Chl}=161,0-4,86 \mathrm{~S}+1,17 \mathrm{P}-0,063 \text { org } \mathrm{Fl}
$$

Es zeigt sich somit also, daß zur Beschreibung der Chlorophyllverteilung die Einbeziehung des Phytoplanktons überhaupt nicht notwendig ist, was aber nicht bedeutet, daß der oben angeführten Beziehung zwischen Chlorophyll und 
Phytoplankton keine Realität zukommt. Bei einer Mehrfachkorrelation ist es vielmehr so, daß die Bindung an andere Komponenten weitaus enger ist und das diese in ihrer Verteilung mit der des Phytoplanktons weitgehend identisch sind. Wird zusätzlich außerdem das Phytoplankton berücksichtigt, so ergibt sich für die Mehrfachkorrelation nur eine bedeutungslose Verbesserung, die in ihrem Wert durch den Verlust eines Freiheitsgrades mehr als ausgeglichen wird:

$$
\mathrm{R}_{\text {Chl.S, P, org Fl, Phytopl }}=0,7439
$$

Betrachten wir jetzt die partiellen Korrelationskoeffizienten, so läßt sich wiederum die geringe Bedeutung des Phytoplanktons nach Eliminierung der Hauptkomponenten aufzeigen:

$$
\begin{array}{ll}
\text { rohl, S.P, org } \mathrm{Fl} & =-0,670 \\
\text { rChl, P.S, org } \mathrm{Fl} & =+0,466 \\
\text { rChl, org Fl.S, P } & =-0,424 \\
\text { rCh, Phytopl.S, }{ }^{p} \text {, org F1 } & =+0,021
\end{array}
$$

Wahrscheinlichkeit

$$
\begin{aligned}
& >99,9 \% \\
& >95 \% \\
& >95 \% \\
& \ll 95 \%
\end{aligned}
$$

Die Korrelation mit dem Salzgehalt berücksichtigt wegen der weitgehenden Identität dieser Messungen gleichzeitig die Temperatur und die Tiefenverteilung.

Die enge Bindung des Chlorophylls zum Phosphor läßt sich vielleicht so erklären, daß aus den organischen, chlorophyllhaltigen Partikeln Phosphat in Freiheit gesetzt wird.

Die negative Beziehung zwischen Chlorophyll und organismischer Fluoreszenz ist bekannt (Kalle, persönliche Mitteilung). Es wäre denkbar, daß die fluoreszierenden Stoffe aus dem Detritus erst bei Zerfall des Chlorophylls in Freiheit gesetzt werden, jedoch muß man mit derartigen Überlegungen sehr vorsichtig sein. Es kann natürlich auch möglich sein, daß Chlorophyll und organismische Fluoreszenz über eine nicht bestimmte Meßgröße indirekt miteinander verbunden sind, lassen sich doch in diesem Falle $\mathrm{S}$ und org Fl auch zusammen durch die Fluoreszenz ersetzen, jedoch erschien der tatsächlich berücksichtigte Zusammenhang wahrscheinlicher.

Abb. 1 zeigt die Beziehung zwischen den gemessenen und den berechneten Chlorophyllwerten a mit Hilfe des Phytoplanktons und b unter Berücksichti-

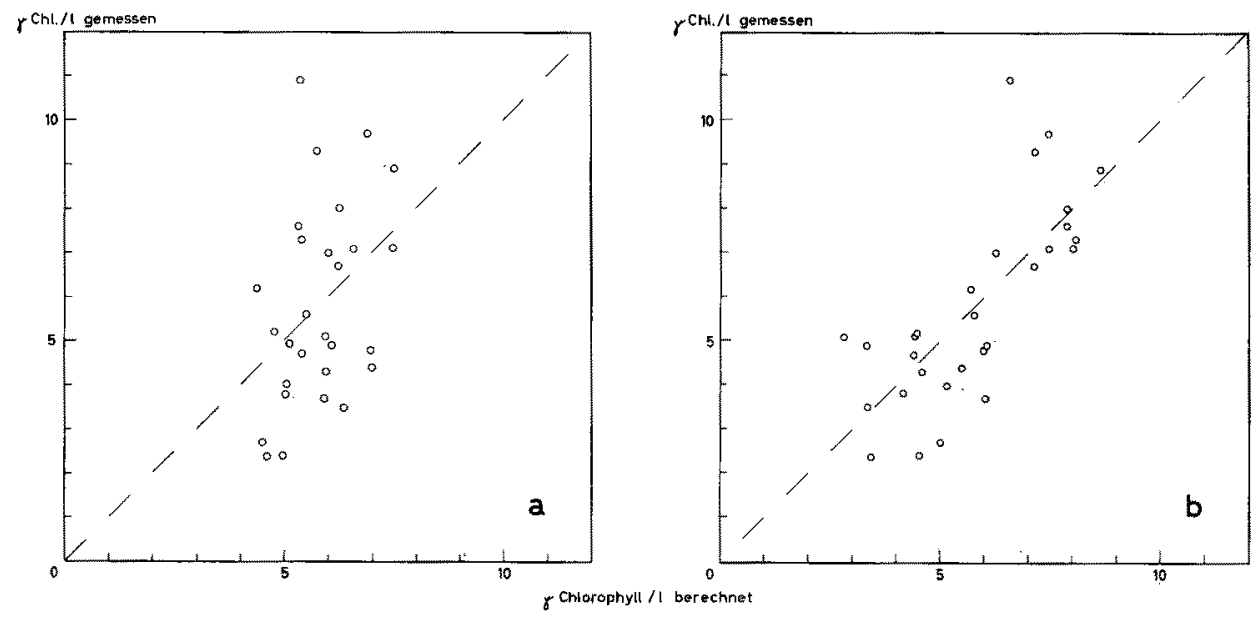

Abb. 1. Beziehung zwischen gemessenem und berechnetem Chlorophyllgehalt:

a: Mit Hilfe des Phytoplanktons und b: Unter Berücksichtigung von Salzgehalt, Phosphat und organismischer Fluoreszenz 
gung von Salzgehalt, Phosphatgehalt und organismischer Fluoreszenz. Die Überlegenheit der multiplen Korrelation ist unverkennbar.

Die Fluoreszenz erweist sich als in erster Linie nur mit dem Salzgehalt korreliert:

$$
\mathrm{rFLLS}=-0,819 \quad \text { Wahrscheinlichkeit } \gg 99,9 \%
$$

Sie ist also offenbar rein terrigen, wie sich aus der Gleichung ergibt:

$$
\mathrm{Fl}=122,2-3,454 \mathrm{~S} \text {, }
$$

denn für Fluoreszenz $=0$ errechnet sich so ein Salzgehalt von $35,4 \%$.

Wie weiter unten (S. 246) deutlich wird, ist die Fluoreszens methodisch mit der organismischen Fluoreszenz verbunden. Jedoch bewirkt die Berücksichtigung dieser Tatsache wegen der ohnehin engen Beziehung Fluoreszenz-Salzgehalt keine entscheidende Verbesserung:

$$
\mathrm{R}_{\mathrm{Fl} \text {. } \mathrm{s} \text {, org FI }}=0,888
$$

Ähnlich liegen die Verhältnisse beim Gelbst off, der auch weitgehend mit dem Süßwasser in die Deutsche Bucht gelangt:

$$
\begin{aligned}
& \mathrm{r}_{\text {Gelbst.S }}=-0,564 \quad \text { Wahrscheinlichkeit }>99 \% \\
& \text { Gelbst }=100,5-2,839 \mathrm{~S} \\
& \text { Gelbst }=0 \quad \text { bei } 35,4 \%
\end{aligned}
$$

Hier läßt sich jedoch durch Einbeziehung weiterer Komponenten eine Verbesserung erzielen:

$R_{\text {eelbst.S, Phytopl, Rest }}=0,701 \quad$ Wahrscheinlichkeit $>99,9 \%$

Gelbst $=109,0-3,077 \mathrm{~S}-0,049$ Phytopl $+0,044$ Rest

Die zugehörigen partiellen Korrelationskoeffizienten lauten:

$$
\begin{aligned}
& \text { rGelbst, S.Phytopl, Rest }=-0,565 \\
& \text { rGelbst, Phytopl.S, Rest }=-0,440 \\
& \text { rGelbst, Rest.S, Phytopl }=+0,376
\end{aligned}
$$

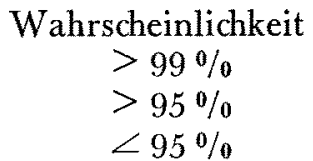

Ob der Gelbstoff aus dem „Rest“ in Freiheit gesetzt wird oder gar durch die Tätigkeit des Phytoplanktons verschwindet, läßt sich so leicht nicht entscheiden.

Noch schwieriger liegen die Dinge beim Kalzium, das zweckmäßig als Funktion von Fluoreszenz, Rest und Phytoplankton beschrieben wird:

$$
\begin{aligned}
& \mathrm{Ca}=419,3-2,57 \mathrm{Fl}-0,266 \text { Rest }+0,235 \text { Phytopl } \\
& \mathrm{R}_{\text {Ca.Fl, Rest, } \text { Phytopl }}=0,625 \quad \text { Wahrscheinlichkeit }>99,9 \%
\end{aligned}
$$

Die partiellen Korrelationskoeffizienten lauten dann:

$$
\begin{aligned}
& r_{C a, \text { Fl.Rest, Phytopl }}=-0,435 \\
& r_{\text {Ca, Rest.Fl, Phytopl }}=-0,426 \\
& r_{\text {Ca, Phytopl.Fl, Rest }}=+0,408
\end{aligned}
$$

$$
\begin{gathered}
\text { Wahrscheinlichkeit } \\
>95 \% \\
>95 \% \\
>95 \%
\end{gathered}
$$

Das Kalzium ist an die Fluoreszenzstoffe und an den Rest wahrscheinlich z. T. so fest komplex gebunden, daß es mit der benutzten Methode nicht erfaßt werden kann. Ferner ist eine Bindung an den Gelbstoff möglich, aber wegen der weitgehenden Identität von Gelbstoff- und Fluoreszenzmessungen nicht sicher nachweisbar. Nach den Beobachtungen beim Gelbstoff wäre es denkbar, daß das Phytoplankton auf die gelösten organischen Stoffe einwirkt und dadurch $\mathrm{Ca} "$ in Freiheit setzt.

Die Phosphatverteilung läßt sich hinreichend mit Hilfe von Fluoreszenz und Chlorophyll angeben, wobei zu beachten ist, daß mit der Fluoreszenz zugleich bis zu einem gewissen Grade auch die Abhängigkeit von Temperatur, Salzgehalt, Tiefe und organismischer Fluoreszenz berücksichtigt wird, da zwi- 
schen diesen Größen ein enger Zusammenhang besteht. Für die Mehrfachkorrelation gilt dann:

$$
\begin{aligned}
& P=5,46-0,413 \mathrm{Fl}+0,182 \mathrm{Chl} \\
& \mathrm{R}_{\mathrm{P} . \mathrm{Fl}, \mathrm{Chl}}=0,577
\end{aligned}
$$

Die partiellen Korrelationskoeffizienten sind:

$$
\begin{aligned}
& \mathrm{rP}, \text { Fl.Chl }=-0,565 \\
& \mathrm{rP, \text {Chl.FI }}=+0,486 \\
& \text { Wahrscheinlichkeit } \\
& >99 \% \\
& >99 \%
\end{aligned}
$$

Es wäre denkbar, daß das Phosphat $z$. T. von den Fluoreszenzstoffen gebunden und dadurch der Bestimmung entzogen wird, woraus dann eine hohe negative Korrelation resultiert. Wo viel Chlorophyll ist, das zumeist an Detritus gebunden ist, hat man mit stärkerem Remineralisationsvorgängen, d. h. mit Freisetzung von Phosphat zu rechnen.

Feintrübung und $\triangle \mathrm{EK}$ lassen sich am besten nur mit der Tiefe korrelieren, d. h., es handelt sich bei den trübenden Stoffen in erster Linie um aufgewirbelte Sedimente.

$$
\begin{aligned}
& \mathrm{r} \Delta \text { EK.m }=+0,935 \\
& \text { rFeintr.m }=+0,716
\end{aligned}
$$$$
\text { Wahrscheinlichkeit }
$$$$
\begin{aligned}
& \gg 99,9 \% \\
& \gg 99,9 \%
\end{aligned}
$$

Die organische Substanz erweist sich als abhängig von Tiefe (zugleich bis zu einem gewissen Grade Temperatur, Salzgehalt und Trübung berücksichtigend), Bakterien, Phytoplankton und Zooplankton:

$$
\begin{aligned}
& \mathrm{C}=0,515-0,0180 \mathrm{~m}+0,0029 \text { Bakt }+ \text { 0,0094 Phytopl - 0,0057 Zoopl } \\
& R_{C . m, \text { Bakt, Phytopl, Zoopl }}=0,665 \quad \text { Wahrscheinlichkeit }>99,9 \%
\end{aligned}
$$

Für die partiellen Korrelationskoeffizienten erhalten wir:

$$
\begin{aligned}
& \text { rC, m.Bakt, Phytopl, Zoopl }=-0,479 \\
& \text { rC, Bakt.m, Phytopl, Zoopl }=+0,494 \\
& \text { ro, Phytopl.m, Bakt, Zoopl }=+0,347 \\
& \text { rC,Zoopl.m, Bakt, Phytopl }=-0,520
\end{aligned}
$$

$$
\begin{aligned}
& \text { Wahrscheinlichkeit } \\
& \begin{array}{c}
>95 \% \\
>95 \% \\
<95 \% \\
>99 \%
\end{array}
\end{aligned}
$$

Die Deutung dieser Befunde ist nicht einfach. Der Kohlenstoff ist in der salzarmen Oberschicht (also im Küstenwasser) zu Hause, das salzreiche Atlantikwasser enthält ja bekanntlich viel weniger C (DuUrsma, 1960). Die organische Substanz scheint z. T. vom Phytoplankton abgegeben zu werden, während sie mit dem Zooplankton stark negativ korreliert ist. Ob sich dies mit Pütters Theorie (PüTTER, 1909) erklären läßt, ob die Tiere weitgehend die organischen Partikel aufzehren, oder ob eine dritte Komponente diese Beziehung nur vortäuscht, kann auf Grund dieser einen Meßreihe nicht entschieden werden. Die Bakterien könnten vielleicht bei höheren C-Konzentrationen bessere Entwicklungsbedingungen haben, was die positive Korrelation erklären würde.

Die Bakterien ihrerseits sind neben dem Nahrungsangebot (Kohlenstoff) von der besiedelbaren Oberfläche abhängig, die in gewisser Weise über die Partikelzahl mit der Trübung identisch sein dürfte.

$$
\begin{aligned}
& \text { Bakt }=-24+569 \triangle \mathrm{EK}+58,6 \mathrm{C} \\
& \mathrm{R}_{\text {Bakt. } \Delta \mathrm{EK}, \mathrm{C}}=0,688 \quad \text { Wahrscheinlichkeit }>99,9 \%
\end{aligned}
$$

Für die partiellen Korrelationskoeffizienten finden wir:

$$
\mathrm{rBakt}, \Delta \mathrm{EK} . \mathrm{C}=+0,671
$$$$
\mathrm{rBakt}_{\mathrm{B}, \mathrm{C} . \Delta \mathrm{EK}}=+0,404
$$

Wahrscheinlichkeit

$$
\begin{aligned}
& >99,9 \% \\
& >95 \%
\end{aligned}
$$

Wir haben es hier also mit dem nicht so seltenen Fall zu tun, daß die Kor- 
relation zwischen Bakterien und Trübung nach Eliminierung des Kohlenstoffs nicht wesentlich von der Mehrfachkorrelation verschieden ist. Die Korrelation ohne Berücksichtigung des Kohlenstoffs ist jedoch eindeutig schlechter:

$$
\text { raakt. } \triangle \mathrm{EK}=+0,609
$$

Die organismische Fluoreszenz ist (wohl weitgehend methodisch bedingt) von der Fluoreszenz und außerdem vom Zooplankton abhängig:

$$
\begin{aligned}
& \operatorname{org~Fl}=74,0-5,44 \mathrm{Fl}+0,126 \text { Zoopl } \\
& \operatorname{Rorg}_{\text {org Fl, Zoopl }}=0,619 \quad \text { Wahrscheinlichkeit }>99,9 \%
\end{aligned}
$$

Die partiellen Korrelationskoeffizienten sind:

$$
\begin{aligned}
& r_{\text {org Fl, Fl.Zoopl }}=-0,580 \\
& \text { rorg Fl, Zoopl.Fl }_{\text {or }}=+0,416
\end{aligned}
$$

$$
\begin{gathered}
\text { Wahrscheinlichkeit } \\
>99 \% \\
>95 \%
\end{gathered}
$$

Es scheint also ziemlich sicher zu sein, daß die organismische Fluoreszenz vom Zooplankton abgegeben wird. Zum Phytoplankton und zu den Bakterien besteht eine derartige Beziehung nicht.

Die Verteilung des Phytoplanktons läßt sich am besten durch folgende Formel beschreiben:

$$
\begin{aligned}
& \text { Phytopl }=-229,2+17,21 \mathrm{t}-3,48 \text { Gelbst }+0,38 \mathrm{~m} \\
& \text { Rehytopl.t, Gelbst, } \mathrm{m}=0,665 \quad \text { Wahrscheinlichkeit }>99,9 \%
\end{aligned}
$$

Als partielle Korrelationskoeffizienten erhalten wir dann:

$$
\begin{aligned}
& \text { rPhytopl, t.Gelbst, } m=+0,629 \\
& \text { rPhytopl, Gelhst.t, }=-0,414 \\
& \text { rPhytopl, m.t, Gelbst }=+0,306
\end{aligned}
$$

$$
\begin{gathered}
\text { Wahrscheinlichkeit } \\
>99,9 \% \\
>95 \% \\
<95 \%
\end{gathered}
$$

In welchem Umfange die Verteilung des Phytoplanktons direkt von der Temperatur oder von der hiermit etwa identischen Verteilung des Salzgehalts abhängt, dürfte kaum zu entscheiden sein. Eine andere Frage ist es nunmehr, ob nicht der Gelbstoff in seiner Verteilung vom Phytoplankton abhängt, so daß die oben gebrachte Beziehung wenig sinnvoll ist, da in ihr das Phytoplankton als abhängig vom Gelbstoff beschrieben wird. Gegen eine solche Auffassung sprechen zwei Gründe:

1. Wir wissen einstweilen nicht zuverlässig, welche Abhängigkeiten in Wahrheit bestehen, können also zunächst nur die Tatsachen feststellen.

2. Ein wichtiges Problem bei dieser Untersuchung ist, in welchem Umfange sind verschiedene Messungen identisch, können sich also gegenseitig ersetzen. Bei dieser Fragestellung ist die Richtung der Abhängigkeit ohnehin ohne Interesse.

Für das Zooplankton finden wir folgenden Zusammenhang:

Zoopl $=-56,0+1,18$ Rest $+1,23$ org Fl $-23,02 \mathrm{C}+6,51 \mathrm{Fl}$

$\mathrm{R}_{\text {Zoopl.Rest, org F1, } \mathrm{C}, \mathrm{Fl}}=0,632$ Wahrscheinlichkeit $>99,9 \%$

Für die partiellen Korrelationskoeffizienten ergibt sich:

$$
\begin{aligned}
& \text { rZoopl, Rest. org Fl, , Fl }=+0,421 \\
& \text { rZoopl, org Fl. Rest, , Fl }=+0,420 \\
& \text { rZoopl, C. Rest, org Fl, Fl }=-0,328 \\
& \text { rZoopl, Fl. Rest, org Fl, }=+0,236
\end{aligned}
$$

$$
\begin{aligned}
& \text { Wahrscheinlichkeit } \\
& >95 \% \\
& >95 \% \\
& <95 \% \\
& \ll 95 \%
\end{aligned}
$$

Das Zooplankton ist offenbar nicht nur - wie schon oben (S. 246) gezeigt - mit der organismischen Fluoreszenz, sondern auch mit dem Rest verbunden. $\mathrm{Ob}$ es diese organischen Partikel als Nahrungsquelle ausnutzt oder durch Zerstörung von Phytoplankton erzeugt, oder ob alles beides der Fall ist, läßt sich 
so nicht entscheiden. Ich möchte auf Grund anderer Beobachtungen (GillBRICHT, 1961) die Nahrungsquelle annehmen.

Der negative Zusammenhang mit dem Kohlenstoff ist nur gering, wird aber bei Eliminierung anderer Komponenten wesentlich größer (S. 245).

Die Fluoreszenz wurde in erster Linie einbezogen, weil die organismische Fluoreszenz weitgehend von ihr abhängig ist. Der geringe Zusammenhang zwischen Zooplankton und Fluoreszenz beweist, daß (nach Eliminierung der übrigen Komponenten) das Zooplankton weder sonderlich an einen Wasserkörper gebunden ist, noch eine ausgeprägte Vertikalverteilung zeigt, denn die Fluoreszenz ist in ihrer Verteilung weitgehend von $\mathrm{S}$ und $\mathrm{m}$ abhängig.

Das Vorkommen des Rests wird zweckmäßig durch Temperatur und Kalzium beschrieben:

$$
\begin{aligned}
& \text { Rest }=91,0+7,06 \mathrm{t}-0,494 \mathrm{Ca} \\
& \begin{array}{l}
\text { Rest. } \mathrm{t}, \mathrm{Ca}=0,626 \\
\text { Wahrscheinlichkeit }>99,9 \%
\end{array}
\end{aligned}
$$

Die partiellen Korrelationskoeffizienten sind:

$$
\begin{array}{lc} 
& \text { Wahrscheinlichkeit } \\
\text { rRest, t. } \mathrm{Ca}=+0,498 & >99 \% \\
\text { rRest, Ca.t }=-0,362 & <95 \%
\end{array}
$$

Der Rest scheint also weitgehend an das warme, salzarme Oberflächenwasser gebunden zu sein. Auf den Zusammenhang mit dem Kalzium wurde schon oben (S. 244) hingewiesen.

\section{Diskussion der Ergebnisse}

Ein Teil der Befunde ist auf jeden Fall weitgehend an Ort und Jahreszeit gebunden: das Fehlen der Sprungschicht und die lineare Zunahme von $\triangle \mathrm{EK}$ und Feintrübung bis zum Boden. Die Abhängigkeit zwischen Fluoreszenz bzw. Gelbstoff und Salzgehalt ist wohl nur in Rand -und Nebenmeeren so ausgeprägt. Die Verteilung der Bakterien dürfte vielleicht grundsätzlich die hier beobachtete Abhängigkeit zeigen. $\mathrm{Ob}$ bei den übrigen Komponenten stets qualitativ ähnliche Verhältnisse vorliegen, muß erst noch untersucht werden. In großen Zügen dürfte dies wohl der Fall sein, in den Einzelheiten ist aber sicher noch mit Uberraschungen zu rechnen, so daß zunächst von theoretischen Erörterungen weitgehend abgesehen werden soll. Wie sehr die angeführten Beziehungsgleichungen nur für die untersuchte Wassermasse zutreffend sind, läßt sich am einfachsten durch Betrachten der konstanten Glieder ermitteln, die oft genug völlig unsinnige Werte ergeben; z. B. würde sich so für das Süßwasser fast Siedetemperatur errechnen. Extrapolationen dieser Art haben eben keinen Sinn, die Gleichungen beschreiben nur die Verhältnisse im wirklichen Beobachtungsbereich, so daß wir allenfalls noch geringe Aussagen über das Ozeanwasser machen können (Fluoreszenz, Gelbstoff).

Die bei Mehrfachkorrelation verbleibende Streuung der Meßdaten um die Gerade (Abb. 1, Tab. 1) entspricht durchweg den Erwartungen. Die methodische Streuung kann nicht ganz erreicht werden, da sich die Verteilung kaum zu $100 \%$ beschreiben läßt, und auch die unabhängigen Variablen Meßfehler aufweisen. Beim Phytoplankton und beim Rest scheint allerdings von einer zu großen Unsicherheit der Zählungen ausgegangen worden zu sein.

Bei den partiellen Korrelationskoeffizienten hängt das Ergebnis naturgemäß weitgehend auch von den eliminierten Größen ab, wie sich dies für die 


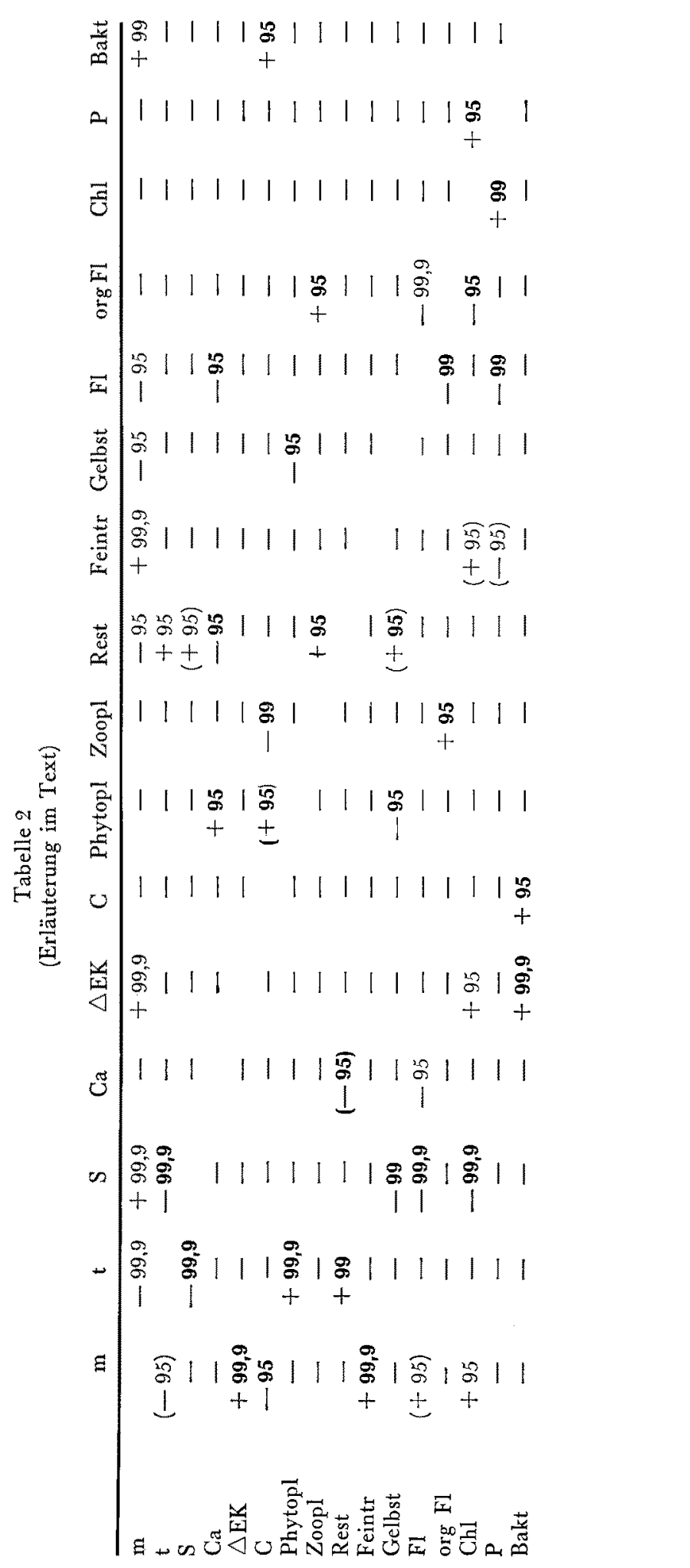


angeführten Beziehungen Ca-Rest (S. 244 und 247) zeigen läßt. Die praktische Auswertung ist daher nicht so objektiv, wie dies zunächst den Anschein hat. Wurde doch schon beim Chlorophyll (S. 243) darauf hingewiesen, daß S + org Fl praktisch mit der Fluoreszenz identisch sind und daher wahlweise benutzt werden können. Es ist dann rein auf Grund der Rechnung nicht zu sagen, welcher Kombination der Vorzug zu geben ist. Ahnlich liegen die Dinge auch, wenn wir Temperatur, Salzgehalt und Fuoreszenz betrachten, die sehr eng korreliert sind. Es ist in diesem Falle nicht möglich, durch Eliminierung einer Größe zuverlässig die Abhängigkeit von einer anderen zu ermitteln. Sowohl der Ansatz bei den Rechnungen als auch die Auswertung der Ergebnisse sind daher nicht frei von den Voraussetzungen, von denen der Beobachter ausgeht.

Dies ist auch bei Tabelle 2 zu beachten, die den Zusammenhang zwischen den verschiedenen Messungen bringt. Hierbei wird von den partiellen Korrelationskoeffizienten ausgegangen, indem jeweils die zuvor zur Beschreibung der Verteilung der einzelnen (vor den Waagerechten stehenden) Komponenten benutzten Messungen eliminiert werden:

$$
\begin{array}{ll}
\mathrm{m} & =\mathrm{f}(-) \\
\mathrm{t} & =\mathrm{f}(\mathrm{S}) \\
\mathrm{S} & =\mathrm{f}(\mathrm{t}) \\
\mathrm{Ca} & =\mathrm{f}(\mathrm{Fl}, \text { Rest, Phytopl) } \\
\triangle \mathrm{EK} & =\mathrm{f}(\mathrm{m}) \\
\mathrm{C} & =\mathrm{f}(\mathrm{m}, \text { Bakt, Phytopl, Zoopl) } \\
\text { Phytopl } & =\mathrm{f}(\mathrm{t}, \text { Gelbst, m) } \\
\text { Zoopl } & =\mathrm{f}(\text { Rest, org Fl, C, Fl }) \\
\text { Rest } & =\mathrm{f}(\mathrm{t}, \mathrm{Ca}) \\
\text { Feintr } & =\mathrm{f}(\mathrm{m}) \\
\text { Gelbst } & =\mathrm{f}(\mathrm{S}, \text { Phytopl, Rest }) \\
\text { Fl } & =\mathrm{f}(\mathrm{S}) \\
\text { org Fl } & =\mathrm{f}(\mathrm{Fl}, \text { Zoopl }) \\
\text { Chl } & =\mathrm{f}(\mathrm{S}, \mathrm{P}, \text { org Fl }) \\
\mathrm{P} & =\mathrm{f}(\text { Fl, Chl }) \\
\text { Bakt } & =\mathrm{f}(\triangle \mathrm{EK}, \mathrm{C})
\end{array}
$$

+ bedeutet dann eine positive Beziehung und - eine negative. Die Zahl dahinter gibt die Wahrscheinlichkeit der Abhängigkeit im angegebenen Sinne in Prozent an. Werden 95\% nicht ganz erreicht, so steht der Wert in Klammern. Ein Strich zeigt geringere Korrelation an. Die schon früher gebrachten partiellen Beziehungen zwischen den verschiedenen Komponenten und den zur Beschreibung ihrer Verteilung benutzten sind fett gedruckt.

Außer den in den Beziehungsgleichungen berücksichtigten (und in der Tabelle fett gedruckten) Bindungen sind enge Korrelationen recht selten. Eine Ausnahme macht hierin nur die Tiefe der Probennahme, die schlechterdings nicht als Funktion einer anderen Messung beschrieben werden kann. Mehrere Komponenten zeigen unter dieser Bedingung eine ausgesprochene Vertikalverteilung. Ansonsten sei als bemerkenswertes Beispiel nur das Chlorophyll erwähnt, das nach Eliminierung von Salz, Phosphatgehalt und organismischer Fluoreszenz mit der Tiefe (und mit der hiermit etwa identischen Trübung) zunimmt. Diese zunächst überraschende Tatsache ist wohl so zu erklären, daß das Chlorophyll sich in seiner Menge nicht so stark mit der Tiefe ändert wie das Salz, so daß die Eliminierung von S eine Umkehr der direkt vorliegenden Ver- 
hältnisse bedingt. Eine zusätzliche Einbeziehung der Tiefe bei der Mehrfachkorrelation bringt jedoch keine nennenswerte Verbesserung, weshalb davon abgesehen wurde. Es ist sehr die Frage, ob spätere Messungen alle Befunde bestätigen werden. Die vorliegenden Betrachtungen können nur einen ersten Eindruck vermitteln und bedürfen zu ihrer Erhärtung weiterer Untersuchungen.

\section{Zusammenfassung}

1. Bei Helgoland wurden im August 1959 auf einer Dauerstation zahlreiche Untersuchungsverfahren parallel angewandt.

2. Die statistische Auswertung ergab komplizierte Abhängigkeiten zwischen den verschiedenen Messungen. Es erwies sich durchweg als notwendig, jeweils eine Komponente in ihrer Verteilung als abhängig von mehreren anderen zu betrachten. Hieraus folgt also, daß nur in seltenen Fällen die Möglichkeit besteht, aus einem Meßergebnis auf eine nicht bestimmte Größe rückzuschließen. Z. B. läßt sich in diesem Falle die Phytoplanktonverteilung nicht befriedigend durch die des Chlorophylls beschreiben.

3. Es ist noch nicht zu übersehen, welche Beziehungen grundsätzliche Bedeutung haben, welche regional und welche zeitlich bedingt sind, und welche nur zufällig gefunden wurden. Fluoreszenz und Gelbstoff dürften aus dem Süßwasser stammen; die organismische Fluoreszenz scheint vom Zooplankton abgegeben zu werden; die Trübung besteht in dem flachen Gezeitenmeer weitgehend aus aufgewirbelten Sedimenten; und die Bakterien sind positiv an Trübung (besiedelbare Partikel) und organische Substanz gebunden.

\section{Summary}

1. In August 1959 a station off Helgoland undertook to determine parallels between a great quantity of components in several vertical series during one day.

2. The statistical treatment gave very complicated connections between the different measurements. Usually it was necessary to regard the distribution of one component as being dependent upon some others. That means there is only in a few cases the possibility of drawing a conclusion from one measurement of a component not determined. It is, for instance, not at all possible in this case to give a good description of the distribution of the phytoplankton by means of the chlorophyll measurements.

3. At the moment one should not overlook which relations have a fundamental meaning, which are affected by region or time and which relations are detected only by chance. Fluorescence and Gelbstoff (Kalle) should be connected with the fresh water; the organismic fluorescence (Kalle) seems to be given off by the zooplankton; the turbidity exists in the flat sea with strong tidal currents in the first range of turned-up sediments, while the bacteria are positively connected with the turbidity (settled particles) and with the organic substances.

\section{Schriftum}

Duursma, E., 1960: Dissolved Organic Carbon, Nitrogen and Phosphorus in the Sea. Dissertation, Amsterdam. 
Gillbricht, M., 1952: Untersuchungen zur Produktionsbiologie des Planktons in der Kieler Bucht I. Kieler Meeresf. B, 2.

- 1957: Ein Verfahren zum oxydativen Nachweis von organischer Substanz im Seewasser. Helgoländ. Wiss. Meeresunters. 6, 1 .

- 1959: Das Phytoplankton im nördlichen Nordatlantischen Ozean im Spätwinter und Spätsommer 1958. Dtsch. Hydrogr. Ztschr. Erg.H. B, 3.

- 1961: The Distribution of Microplankton and Organic Matter in the Northern North Atlantic during Late Winter and Late Summer 1958. Rapports et Procès-Verbeaux. (Im Druck.)

Joseph, J., 1959: Die Trübungsverhältnisse in der Irminger See im Juni 1955 und ihre hydrographischen Ursachen. Ber. Dt. Wiss. Komm. Meeresforsch. 15, 3.

Kalle, K., 1934: Meereskundlich-chemische Untersuchungen mit Hilfe des Zeiss'schen Pulfrich-Photometers. III. Mitt. Methodische Untersuchung der Phosphatgehalts-Bestimmung. Ann. d. Hydrographie usw. 62.

- 1951: Meereskundlich-chemische Untersuchungen mit Hilfe des Zeiss'schen Pulfrich-Photometers. VII. Mitt. Die Mikrobestimmung des Chlorophylls und der Eigenfluoreszenz des Meerwassers. Dt. Hydrogr. Ztschr. 4.

Krey, J., 1958: Chemical Methods of Estimating Standing Crop of Phytoplankton. Rapports et Procès-Verbeaux. 144

Nordli, E, 1957: Experimental Studies on the Ecology of Ceratia. Oikos 8, 2

Pütter, A., 1909: Die Ernährung der Wassertiere und Der Stoffhaushalt der Gewässer. G. Fischer, Jena.

ZoBel1, C., 1941: Apparatus for collecting water samples from different depths for bacteriological analysis. Journ. Mar. Res. 4.

- 1946: Marnie Microbiology: a monograph on hydrobacteriology. Chronica Botanica Co., Waltham, Mass., p. 41. 